\title{
ORIGINAL
}

\section{ESTIMACIÓN DE LA PREVALENCIA DE HIPOTIROIDISMO EN LLEIDA A PARTIR DE LA PRESCRIPCIÓN DE HORMONAS TIROIDEAS}

M. a Catalina Serna Arnáiz (1), Leonardo Galván Santiago (2), Eduardo Gascó Eguiluz (1), Montserrat Manrique Manrique (1) y Maa Mar Foix Oña, Elisabet Martín Gracia (1)

(1) Área Básica de Salud Eixample, Lleida.

(2) Región Sanitaria de Lleida.

\section{RESUMEN}

Fundamento: El hipotiroidismo es una enfermedad frecuente, que cursa con una sintomatología, en ocasiones inespecífica, lo que dificulta el diagnóstico. El objetivo del estudio es estimar la prevalencia de hipotiroidismo en Lleida a través de las prescripciones de la hormona tiroidea y comparar los resultados con los de otras regiones.

Métodos: Estudio observacional retrospectivo. 2.854 personas que reciben tratamiento con hormona tiroidea mediante receta de $\mathrm{l}$ Seguridad Social. Meses de octubre a diciembre del 2001. Variables: la edad, el sexo y región sanitaria.

Resultados: La prevalencia de hipotiroidismo en el 2001 es de 8,4 casos por 1.000 habitantes día (IC 95\%:9,23-7,47). La edad media es de 60 años (DS<:16,6). La distribución por sexo: 2,4 \%o en hombres y $12,4 \%$ en mujeres. Por grupos de edad: Menores de 15 años un 3,6\%o, entre 15 y 64 años de $6,8 \%$ y en mayores de 64 de 12,5. Las cifras más elevadas se encuentran en las áreas rurales (Seu d'Urgell: $10,5 \%$ ) y las inferiores en áreas urbanas(Eixample:5,8\%, Balafia:5,4\%o)

Conclusiones: La cifra de hipotiroidismo en tratamiento es, como en otros estudios, superior en mujeres y predomina en los mayores de 64 años. Sin embargo, observamos valores de prevalencia inferiores en relación a los datos de un estudio poblacional previo de la misma región, indicando un posible infradiagnóstico de la enfermedad.

Palabras clave: Hipotiroidismo. Hormonas tiroideas. Farmacoepidemiología. Prevalencia.
ABSTRACT

\section{Estimate of Hypothyroidism Prevalence in Lleida, Spain, Based on Thyroid Hormone Prescription}

Background: Hypothyroidism is a common disease sometimes showing no specific symptoms, thus being difficult to diagnose. Thi study is aimed at estimating the prevalence of hypothyroidism in Lleida by way of thyroid hormone prescriptions and comparing the results with those from other regions.

Methods: A retrospective observational study. A total of 2,854 individuals undergoing thyroid hormone treatment through Social Insurance prescriptions. Moths of October-December 2001. Variables: age, sex and healthcare region.

Result: The prevalence of hypothyroidism for 2001 is 8.4 cases per 1000 inhabitants/day (IC 95\%:9.23-7.47). Average age: 60 (DS<:16.6). Age spread:2.4\% among males and $12.4 \%$ among females. By age groups: Under age 15: 3.,6\%o, 15- 64 age group, 6.8 $\% o$ and those over age $64,12.5 \%$. The highest figures were found in the rural areas (Seu d'Urgell:10.5\%o), and the lowest in urban areas (Eixample:5.8\%o, Balafia:5.4\%o)

Conclusions: Hypothyroidism under treatment figure is, as in other studies, higher among females and predominant among those over 64 years of age. We however found prevalence values lower than the data from a prior populational study for the same region, indicating a possible underdiagnosing of this disease.

Key words: Hypothyroidism. Thyroid hormones. Pharmacoepidemiology. Prevalence.
Correspondencia:

M Catalina Serna Arnáiz

C/ Mestre Tonet $30.3^{\circ} 2^{\mathrm{a}}$

25003 Lleida.

Correo electrónico: catserna@ terra.es

\section{INTRODUCCIÓN}

El hipotiroidismo es el síndrome clínico y bioquímico resultante de una disminución de la producción hormonal de la glándula 
tiroides. La frecuencia de hipotiroidismo varia considerablemente dependiendo de la población estudiada. En el estudio longitudinal de Wickham ${ }^{1}$, la prevalencia era de 0,8 $1,1 \%$ siendo la edad de los pacientes de 30 a 76 años. En Lleida Gascó et al $^{2}$ obtienen en 1995 una prevalencia de hipotiroidismo de $3,4 \%$ (IC95\%:1,7-4,2) cifra superior a la obtenida por Vila ${ }^{3}$ en 1990 con una prevalencia del $1,2 \%$.

En todos los estudios se obtiene una prevalencia mayor en mujeres que en hombres y esta diferencia se incrementa con la edad. La prevalencia de hipotiroidismo puede calcularse a partir de la prescripción de hormona tiroidea, dado que es un tratamiento específico, generalmente el empleo del mismo es indicativo de esta patología, aunque los datos que obtendremos serán de hipotiroidismo diagnosticado y tratado ${ }^{4,5}$. En la Autonomía de Cataluña, por un cambio en la normativa vigente, se inició un registro informatizado de las prescripciones y pacientes que acudían a las farmacias, con receta de la Seguridad Social (no se podía dispensar los fármacos sin la presentación de la tarjeta sanitaria).Este hecho nos permite conocer los pacientes que reciben un tratamiento y además la edad y sexo de los mismos, aportando datos novedosos a los estudios de utilización de medicamentos. El objetivo de este estudio es estimar la prevalencia de hipotiroidismo en Lleida a partir de la prescripción de hormona tiroidea y la distribución por grupos de edad y sexo; así como comparar los resultados con los obtenidos en un estudio poblacional, realizado en 1995, en esta población ${ }^{2}$, y con los estudios que estiman la prevalencia a partir de datos de consumo de hormonas tiroideas. El cálculo de la prevalencia se ha realizado tanto por pacientes que reciben hormona tiroidea como por dosis habitante día, a partir de la dosis diaria definida.

\section{SUJETOS Y MÉTODOS}

Se realiza un estudio observacional retrospectivo de las dispensaciones de fármacos del grupo HO3A de la Clasificación Anatómica Terapéutica, realizadas por todas las oficinas de farmacia de la Región Sanitaria de Lleida, con receta de la Seguridad Social. En la tabla 1, están las cinco presentaciones comerciales de hormona tiroidea disponibles en España ${ }^{6}$. El periodo de recogida de datos ha sido durante los meses: octubre, noviembre y diciembre del año 2001 ya que las oficinas de farmacia disponen de tres meses de margen para la facturación de recetas una vez se ha dispensado el medicamento. Debido a que el programa registra a cada paciente una sola vez (aunque acuda cada mes a por la receta a la farmacia), al cabo de tres meses, es probable que hayan acudido a buscar la prescripción crónica todos los pacientes y por lo tanto obtengamos su registro.

En el tratamiento de los datos se ha respetado en todo momento la confidencialidad de los individuos participantes.

En las oficinas de farmacia son informatizados los datos siguientes de las recetas: $\mathrm{La}$ Unidad productiva (o Área Básica de Salud) en la que se entrega la receta. Los datos del paciente y el código de identificación personal (CIP) del cual se obtiene la edad y sexo del paciente y el fármaco prescrito. Así las variables de estudio son: edad, sexo de los pacientes con tratamiento tiroideo y lugar de prescripción de la Región Sanitaria de Lleida. Se ha realizado una agrupación de resultados por Áreas Básicas de Salud o Unidades Productivas. Incluye algunas de carácter urbano: Eixample, Balafia y Bordeta (pertenecientes a Lleida ciudad) y el resto que son de carácter rural en su mayoría. Así mismo, se ha calculado la dosis habitante día (DHD) según las técnicas del Drug Utilization Research Group (DURG) ${ }^{7}$, a parir de la Dosis diaria definida/1.000 habitantes. La Dosis Diaria Definida (DDD) se define como la dosis media diaria habitual de un medicamento cuando se utiliza para su indicación principal en adultos; en el caso de la hormona tiroidea es de 150 microgramos ${ }^{7}$. 
Tabla 1

Especialidades farmacéuticas de hormona tiroidea disponibles en España en el año 2001

\begin{tabular}{|l|c|c|}
\hline Presentación comercial & Comp osición & $\mathbf{N}^{\mathbf{0}}$ de unidades por envase \\
\hline Dexnon & $100 \mathrm{mcg}$ & 100 comprimidos \\
Tiroides Leo I & $0,1 \mathrm{mg}$ & 50 grageas \\
Tiroides Leo II & $0,2 \mathrm{mg}$ & 50 grageas \\
Tiroides Leo IV & $0,4 \mathrm{mg}$ & 50 grageas \\
Levothroid & $100 \mathrm{mcg}$ & 50 comprimidos \\
Levothroid & $50 \mathrm{mcg}$ & 50 comprimidos \\
Levothroid & $500 \mathrm{mcg}$ & Vial inyectable \\
Tiroxina Leo & $100 \mathrm{mcg}$ & 50 comprimidos \\
Thyrax & $100 \mathrm{mcg}$ & 50 comprimidos \\
Thyrax & $25 \mathrm{mcg}$ & 50 comprimidos \\
\hline
\end{tabular}

Los datos de población los hemos obtenido del padrón de habitantes del año 1996 y actualizado en el año 1999. El cálculo de la prevalencia se realiza calculando el cociente entre personas que reciben hormona tiroidea en una zona partido por la población correspondiente a esa zona; ya sea; de cada unidad productiva, comarca o de toda la Región Sanitaria. Las variables cualitativas se expresan en proporciones y las cuantitativas mediante media y desviación estándar, ambas con sus correspondientes intervalos del $95 \%$.

\section{RESULTADOS}

La prevalencia de hipotiroidismo en tratamiento con levotiroxina en la provincia de Lleida es de 8,4 por mil habitantes (IC95\%:7,4-9,2) en el 2001. En números absolutos son 2854 pacientes en tratamiento. La edad media es de 60 años (DS:16,6). En la distribución por sexos presentan una prevalencia de 2,4 por mil los hombres y las mujeres de 12,4 por mil. Por grupos de edad la prevalencia en menores de 15 años es de 0,5 por mil entre 15 y 64 años de un 6,8 por mil y en los mayores de 64 de 12,5 por mil. En la tabla 2 presentamos la distribución por edad y sexo. La mayor prevalencia se encuentra en el grupo de mujeres de 15 a 64 años con un 36 por mil. En la tabla 3 se encuentran los resultados de los pacientes en tratamiento con hormona tiroidea por unidades productivas o Áreas Básicas de Salud. Las cifras de prevalencia oscilan entre un 5 y un 10 por mil. Las cifras más altas halladas son las de Seu d'Urgell (10,5 por mil) y en Alt Urgell Sud con un 13 por mil y las más bajas son las de Eixample 5,8 por mil y Balafia con 5,4 por mil. Estas últimas son Áreas Básicas urbanas. La zona con una edad media de consumo de levotiroxina inferior es la de Bordeta con una media de 50,6 años (DS:10,2) y la más alta la de Afarras con 71 años de media (DS:12,1). El cálculo de la prevalencia de toda la Región Sanitaria por DHD es de 5,39 por mil habitantes.

Tabla 2

Prevalencia (IC95\%) de hipotiroidismo en tratamiento con levotiroxina por grupos de edad

\begin{tabular}{|l|l|l|}
\hline Edad & \multicolumn{1}{|c|}{ Hombres * } & \multicolumn{1}{c|}{ Mujeres * } \\
\hline $0-14$ & $0^{\prime} 36$ & $0^{\prime} 79$ \\
& $\left(0^{\prime} 365-0^{\prime} 354\right)$ & $\left(0^{\prime} 871-0^{\prime} 70\right)$ \\
\hline $15-64$ & $1^{\prime} 8$ & 36 \\
& $\left(1^{\prime} 822-1^{\prime} 77\right)$ & $\left(36^{\prime} 28-35^{\prime} 71\right)$ \\
\hline$>64$ & $5^{\prime} 2$ & $18^{\prime} 3$ \\
& $\left(5^{\prime} 245-5^{\prime} 154\right)$ & $\left(18^{\prime} 6-18^{\prime} 28\right)$ \\
\hline
\end{tabular}

*Porcentaje en tanto por mil. 
Tabla 3

Prevalencia de hipotiroidismo en tratamiento con levotiroxina sódica por Áreas Básicas de Salud

\begin{tabular}{|c|c|c|c|c|}
\hline Población & Prevalencia * & $\mathbf{N}$ & $\begin{array}{c}\text { Edad media } \\
\text { (años) }\end{array}$ & Zona \\
\hline Agramunt & 5 & 33 & 53 & \multirow[t]{16}{*}{ Rural } \\
\hline Alfarras & $8 ' 2$ & 83 & 71'1 & \\
\hline Alt Urgell & 13 & 60 & $59 ' 8$ & \\
\hline Vall d'Aran & $7 ' 8$ & 56 & 54 '9 & \\
\hline Bellpuig & 8 & 51 & 60 & \\
\hline Pallars Sobira & $6 \cdot 5$ & 38 & 59 & \\
\hline Pobla de Segur & $8 ' 6$ & 41 & 57 & \\
\hline Ponts & 6 & 22 & $63 ' 5$ & \\
\hline Tremp & 7'1 & 58 & 59'1 & \\
\hline Alcarras & 677 & 54 & $51 ' 5$ & \\
\hline Almacelles & $7 ' 1$ & 55 & 61 & \\
\hline Alta Ribagorça & $5 ' 9$ & 21 & 60 & \\
\hline Artesa de Segre & $8 ' 1$ & 32 & $66 ’ 25$ & \\
\hline Granadella & $7 ' 6$ & 28 & $63 ' 5$ & \\
\hline Lleida rural sud & $7 ' 4$ & 48 & $61 ’ 2$ & \\
\hline Seros & 6 & 59 & 64'1 & \\
\hline Balaguer & 9'1 & 224 & $58^{\prime} 1$ & \multirow[t]{6}{*}{ Semirural } \\
\hline Cervera & $7 ' 4$ & 110 & $59^{\prime} 2$ & \\
\hline Seu d'urgell & $10^{\prime} 5$ & 152 & $64{ }^{\prime} 4$ & \\
\hline Tarrega & $7 ’ 3$ & 137 & $60 ’ 2$ & \\
\hline Borges Blanques & $9 ' 2$ & 151 & $58 ' 3$ & \\
\hline Pla d'Rrgell & 9'1 & 265 & $50^{\prime} 1$ & \\
\hline Eixample & $5 ' 8$ & 145 & $59^{\prime} 6$ & \multirow[t]{3}{*}{ Urbana } \\
\hline Balafia & $5 ' 4$ & 131 & 58 & \\
\hline Bordeta & 6 & 83 & $50^{\prime} 6$ & \\
\hline
\end{tabular}

\section{DISCUSION}

El estudio de la prevalencia de hipotiroidismo a través de las personas que consumen hormona tiroidea puede llevarse a cabo por el carácter crónico de la enfermedad y por tratarse de una enfermedad que tiene como único tratamiento la hormona tiroidea. Sin embargo, como limitaciones al estudio observamos que los resultados obtenidos nos aportaran datos del hipotiroidismo tratado y no del hipotiroidismo sin diagnosticar ni tratar, por lo que tiende a subestimar la prevalencia real ${ }^{8-10}$. Otra de las limitaciones se debe a que recogemos datos de los pacientes que acuden al sistema sanitario público, pudiendo quedar pacientes sin contabilizar debido a que reciban tratamiento en la asistencia privada. Se considera que este número de pacientes debe ser mínimo debido al pago reducido de medicamentos por parte del usuario, por la Ley General de Sanidad de $1986^{11}$, disminuyendo en gran medida la derivación de pacientes a la asistencia priva- 
da y aproximando el consumo obtenido por los datos del sistema sanitario al consumo real $^{12}$. Tampoco incluiría a los pacientes con cobertura por Muface (el porcentaje de recetas de esta entidad es de un 2,2\% del total de prescripciones de la Región Sanitaria) ni a los que se hallen en régimen de ingreso hospitalario superior a tres meses. Estos porcentajes tampoco consideramos que modifiquen el resultado en gran medida.

En este estudio hemos obtenido una prevalencia del 8,4 por mil habitantes (IC95\%: 7,4-9,2) en la Región Sanitaria de Lleida, utilizando el cálculo de los pacientes con prescripción de hormona tiroidea, y de un 5,4 por mil habitantes con el cálculo de la DHD. Esta diferencia representa que existe un porcentaje de pacientes amplio que no reciben la dosis plena de levotiroxina que es de $150 \mathrm{mcg} / \mathrm{d}$.

Comparando con otros estudios, se recoge una prevalencia más alta que la referida por Morant ${ }^{13}$ con 4,33 casos por 1000 habitantes en España, estudio realizado a partir de las DHD. Es posible que en Lleida se dé una mayor prevalencia por el déficit de yodo padecido por esta población en años anterio$\mathrm{res}^{14}$, ya que la frecuencia de hipotiroidismo aumenta en las zonas con déficit de yodo ${ }^{15}$; De hecho, se observa prevalencias más elevadas en las zonas rurales, con respecto a las áreas urbanas. Las regiones rurales han padecido, sobretodo en los Pirineos, una gran deficiencia nutricional de yodo. En el estudio de prevalencia realizado en Lleida por Gascó ${ }^{2}$ et al. Se encontró una prevalencia de hipotiroidismo de 3,4\% (IC95\%:1,78$4,2)$; siendo el hipotiroidismo clínico del 1,24 . Los pacientes que presentan hipotiroidismo clínico precisan tratamiento con hormonas tiroideas, estos corresponden a un 12,4 por mil habitantes, por lo que se obtiene una prevalencia superior a la que hemos observado en nuestro estudio con un 8,4 por mil. Esta diferencia es probable que sea debida a que se trata de un estudio poblacional, detectando los pacientes sin diagnosti- car y sin tratar, y en nuestro estudio contabilizamos únicamente los pacientes en tratamiento. Estos datos nos evidencian un infradiagnóstico de la enfermedad, aunque también puede deberse a un descenso en la prevalencia después de 7 años, en los que se ha incrementado el aporte de yodo en la población.

Respecto a la distribución por sexos 5,1/1 la relación mujer/hombre. La distribución por sexos es similar a la descrita en otros estudios $^{2,16,17}$.

El hecho de que la sintomatología del hipotiroidismo sea en adultos inespecífica y de instauración insidiosa con síntomas como: fatigabilidad, estreñimiento, entre otros, que son frecuentes en la población general, hacen difícil su sospecha en la práctica clínica ${ }^{18}$. Por ello, el Programa de actividades preventivas y de promoción de la salud recomienda el cribado de mujeres mayores de 50 años con clínica inespecífi$\mathrm{ca}^{19}$. El conocimiento de la prevalencia de hipotiroidismo con tratamiento en nuestro medio, nos puede orientar en la práctica clínica para la búsqueda activa de casos, en personas con factores de riesgo, cuando las cifras de prevalencia de nuestro entorno son inferiores a las esperadas por grupos de edad y sexo. El hecho de que en nuestro estudio observemos cifras inferiores a las obtenidas en el estudio poblacional realizado en la misma zona nos alerta ante un posible infradiagnóstico de la enfermedad. Teniendo presente las consideraciones comentadas respecto al diferente periodo de estudio y a la metodología empleada.

Los estudios de utilización de medicamentos constituyen un importante medio para la evaluación de la prescripción farmacológica y el conocimiento de las enfermedades. Consideramos necesaria la realización de estudios con esta metodología para evaluar las diferencias en el uso de fármacos en distintas regiones y en diferentes tiempos. 


\section{BIBLIOGRAFÍA}

1. Tunbridge W, Evered D, Hall R, Appleton D, Brewis $\mathrm{M}, \mathrm{Clark} \mathrm{F}$ et al. The spectrum of thyroid disease in a community; The Whickham survey. Clin Endocrinol 1977; 7:481-93.

2. Gascó E, Serna MC, Vázquez A, Peremiquel M, Ibarz M, Serra L. Prevalencia del trastorno de la función tiroidea en la provincia de Lleida. Aten Primaria 1999; 24:475-60.

3. Vila Ballester L1. Característiques clíniques i epidemiològiques del goll en la població de la Cerdanya. [Tesis doctoral]. Universitat Autònoma de Barcelona;1993.

4. Iñesta García A. Estudios de utilización de medicamentos en España y análisis de la situación farmacoterapéutica. En: Estudios de utilización de medicamentos. Madrid: Insalud;1992.p.15-48.

5. Figueiras A, Camaño F, Gestal JJ. Metodología de los estudios de medicamentos, en Atención Primaria. Gac Sanit 2000; 14 (supl 3): 7-19.

6. Consejo General de Colegios Oficiales de Farmacéuticos. Catálogo de especialidades farmacéuticas. Madrid: Consejo General de Colegios Oficiales de Farmacéutidcos;1996

7. Nordic Council on Medicines. ATC. Index with DDDs. WHO Colaborating Center for Drug Statistics Methodology. Oslo: WHO;1999

8. Sawin CT, Castelli WP, Hershman JM, McNamara P, Bacharach P. The aging thyroid: Thyroid deficiency in the Framingham Study. Arch Intern Med. 1985;145:1386-8.

9. Hunter I, Greene SA, MacDonal TM, Morris AD. Prevalence and aetiology of hypothyroidism in the young. Arch Dis Child 2000; 83(3):207-10.

10. García Iñesta A. Estudios de utilización de medicamentos en España y análisis de la situación far- macoterapéutica. En: Instituto Nacional de salud, editor. Estudios de utilización de medicamentos. Madrid; Insalud;1992.p.15-48.

11. Ley 14/1986 de 25 de abril, Ley General de Sanidad. BOE núm.112 de 24/4/86

12. Sartor F, Walckiers D. Estimate of disease prevalence using drug consumption data. Am J Epidemiol 1995;141:782-7.

13. Morant Ginestar C, Criado-Álvarez JJ, García-Pina R, Pérez Garrido B. Estimación de la prevalencia de hipotiroidismo en España a partir del consumo de hormonas tiroideas (1996-1999). Rev Esp Salud Pública 2001;75:337-44.

14. Serra i Majem L. Epidemiología del goll endèmic a Catalunya. [Tesi doctoral]. Facultad de Medicina de la Universidad Autónoma de Barcelona;1987.

15. Delange F. The disorders induced by iodine deficiency. Thyroid 1994;4:107-28.

16. Bjoro T, Holmen J, Kruger O, Midthjell K, Hunstad K, Schreiner T et al. Prevalence of thyroid disease, thyroid dysfunction and thyroid peroxidase antibodies in a large, unselected population. The Health Study of Nord-Trondelag (HUNT). Eur J Endocrinol 2000; 143(5) :639-47.

17. Bagchy N, Brown T, Parish F. Thyroid dysfunction in adults over age 55 years. A study in urban us community. Arch Intern Med 1990;150:785-7.

18. Ciruana R. Actividades de prevención en grupos de alto riesgo. En: Programa de actividades preventivas y de promoción de la salud en atención primaria. V Reunión Anual. Red de Centros Investigadores de la SEMFYC, diciembre; 1992.

19. Olloqui Mundet J, Ochoa Prieto J. Medicina de familia en el seguimiento del hipotiroidismo. Aten Primaria 2002;29(1):36-8. 